\title{
The toxic effects and possible mechanisms of Bisphenol $A$ on oocyte maturation of porcine in vitro
}

\author{
Teng Wang ${ }^{1}$, Jun Han ${ }^{1}$, Xing Duan ${ }^{1}$, Bo Xiong ${ }^{1}$, Xiang-Shun Cui ${ }^{2}$, Nam-Hyung Kim ${ }^{2}$, \\ Hong-Lin Liu ${ }^{1}$, Shao-Chen Sun ${ }^{1}$ \\ ${ }^{1}$ College of Animal Science and Technology, Nanjing Agricultural University, Nanjing 210095, China \\ ${ }^{2}$ Department of Animal Sciences, Chungbuk National University, Cheongju 361-763, Korea \\ Correspondence to: Shao-Chen Sun, e-mail: sunsc@njau.edu.cn \\ Keywords: BPA, cytoskeleton, autophagy/apoptosis, oxidative stress, epigenetics \\ Received: January 20, 2016 \\ Accepted: March 28, 2016 \\ Published: April 11, 2016
}

\section{ABSTRACT}

Bisphenol A (BPA) and Di-(2-ethylhexyl) phthalate (DEHP) are widely used in the plastic industry such as water bottles, containers, packaging and toys. BPA and DEHP are shown to be the endocrine disruptors which disturb the endocrine system and are linked to several diseases including infertility. In this study, we investigated the effects of BPA exposure on porcine oocyte maturation and its possible reasons. Our results showed that: (i) the rates of oocyte maturation significantly decreased with $250 \mu \mathrm{M}$ BPA treatment in vitro, but not DEHP. This might be due to the delayed cell cycle progression of oocyte maturation. (ii) BPA treatment resulted in abnormal cytoskeletons on porcine oocytes, showing with aberrant actin distribution, spindle morphology and chromosome alignment, which was further confirmed by the reduced p-MAPK level. (iii) The fluorescence intensity of histone methylation (H3K4me2) and DNA methylation ( $5 \mathrm{mC}$ ) levels were altered after BPA treatment, indicating that epigenetic modification was disturbed. (iv) BPA-exposed oocytes had higher rates of early stage apoptosis/autophagy, and this may be resulted from the increased level of oxidative stress. Collectively, our results indicated that porcine oocytes maturation was disrupted after BPA treatment through disrupting cytoskeletal dynamics, epigenetic modifications and inducing apoptosis/autophagy.

\section{INTRODUCTION}

The growing evidence indicates that environmental contaminants can pose negatively risks to animals and human health. Because some of these substances can mimic and alter the actions of endogenous hormones, some potentially disrupting endocrine function in humans thereby they are referred to as endocrine disruptors (EDC) $[1,2]$. Di(2-ethylhexyl) phthalate (diethyl-hexyl phthalate, DEHP) is widely used as a plasticizer in manufacturing products made by polyvinyl compounds, and it is an estrogen-like chemical $[3,4]$. DEHP is reported as a potent reproductive toxicant and it can cause gonadal morphological or functional alterations in both sexes by acting as endocrine disruptors [5, 6]. Similarly to DEHP, Bisphenol A (BPA) is also a high production volume environmental estrogen-like chemical, and it is widely used in a variety of manufacturing polycarbonate plastic products posing a risk health to human [3]. In 2010,
Canada is the first country that announced BPA is a toxic substance, and the European Union, Canada and the United States have banned BPA in baby bottles.

Some studies have shown that BPA could stimulate prolactin release $[7,8]$, alter thyroid hormone action $[8,9]$, impair aromatase expression [10] and act as an anti-androgen [11]. Human exposure to BPA is nearly ubiquitous and takes place through inhalation, ingestion, and dermal absorption. Females are born with a limited number of oocytes, it is significantly decreased in number from the second trimester of the fetal period until menopause through progressive apoptosis [12]. Recent studies have shown that BPA exposure resulted in developmental genitourinary anomalies, sperm abnormality and sperm DNA damage induced in spermatozoa and epigenetic modifications in off-spring, as well as decreased the epididymal weight and increased prostate weight in male rodents [13-16]. The toxic effects of BPA on oocyte quality have been shown with in vitro 
mouse models [17, 18], in vivo mice models [19-21], in vitro human models [22]. These previous studies have reported that BPA is toxic to fertilization, but most of these studies on oocytes maturation was from clinical aspect and the mechanisms of toxicity are still not fully understood. To find the causes and mechanism for toxic effects of BPA exposure on oocytes, we investigated this through epigenetic modification and apoptosis/autophagy aspects with the porcine model, since the genome of porcine is more close to human species, which could more concisely reflect the reproduction system of human.

Several cellular processes like epigenetic modifications, apoptosis/autophagy and oxidative stress are all critical for oocyte maturation. Apoptosis, a programmed cell death which includes prenatal germ cell death, granulosa cell death during post-natal follicular atresia, plays a major role in the elimination of germ cells at all the stages of oogenesis and ovulation [24, 25]. And autophagy influences maternal mRNA degradation and apoptosis during porcine parthenote development in vitro $[26,27]$. Meanwhile, autophagy is also critical for in vitro maturation and improves the nuclear and cytoplasmic maturation of porcine oocytes [27]. Oxidative stress also inhibited oocyte maturation, and our previous study showed that HT-2 toxin induced oxidative stress inhibited mouse oocyte polar body extrusion [25]. Another report showed that oocytes maturation inhibited by oxidative stress could be protected from melatonin [28]. BPA was shown to induce oxidative stress which resulted in DNA damage in INS-1 cells [29].

Till now there is still little research focused on the influence on porcine oocyte maturation and especially the mechanisms of its toxicity. Therefore, the objective of the present study was to evaluate the influence of acute exposure to BPA and DEHP on porcine oocyte maturation, subcellular structure, epigenetic modification, oxidative stress, autophagy and apoptosis. Our study suggests that BPA disrupts porcine oocytes maturation through changing epigenetic modification, inducing oxidative stress, excessive autophagy and apoptosis.

\section{RESULTS}

\section{BPA but not DEHP treatment results in the failure of polar body extrusion in porcine oocytes in vitro}

We first found that exposure to BPA had immediate impact on the expansion of intact porcine COC cultured in vitro. Porcine COCs were cultured in normal maturation medium for $44 \mathrm{~h}$ in the presence of BPA with different concentrations $(200 \mu \mathrm{M}, 250 \mu \mathrm{M})$ or DEHP $(250 \mu \mathrm{M}$, $500 \mu \mathrm{M}, 750 \mu \mathrm{M}, 1 \mathrm{mM}, 5 \mathrm{mM})$, and then the polar body extrusion rates were examined. As shown in Figure 1A, the good expansion of the peripheral layers of cumulus cells was observed in control maturation group, whereas it was much weaker in BPA treated COCs. Moreover, most of control oocytes had extruded polar bodies and were arrested at the MII stage, whereas in BPA treated oocytes polar body extrusion was suppressed (Figure 1B). BPA treatment resulted in reduced polar body extrusion in a dosedependent manner (Figure 1C). BPA treatment effectively inhibited polar body emission at a concentration of $200 \mu \mathrm{M}$ and $250 \mu \mathrm{M}$. These results showed that $83.67 \pm 2.86 \%(\mathrm{n}=$ 367 COCs) of control oocytes had extruded polar bodies. However, with $200 \mu \mathrm{M}$ and $250 \mu \mathrm{M}$ BPA treatment, the rates of polar body extrusion were significantly reduced to $62.32 \pm 3.39 \%(\mathrm{P}<0.05 ; \mathrm{n}=165 \mathrm{COCs})$ and $43.19 \pm$ $1.38 \%(\mathrm{P}<0.01 ; \mathrm{n}=146 \mathrm{COCs})$. The results suggest that exposure to BPA causes the failure of polar body extrusion in porcine oocytes. However, with $250 \mu \mathrm{M}, 500 \mu \mathrm{M}, 750$ $\mu \mathrm{M}, 1 \mathrm{mM}$ and $5 \mathrm{mM}$ DEHP treatment, the rates of polar body extrusion had no significant variation, as shown in Figure 1D, the rates of polar body were: $81.18 \pm 0.64 \%(\mathrm{n}=$ $463)$ for controls vs. $79.16 \pm 5.17 \%(\mathrm{n}=159)$ for $250 \mu \mathrm{M}$, $79.16 \pm 5.17 \%(\mathrm{n}=177)$ for $500 \mu \mathrm{M}, 76.39 \pm 2.93 \%(\mathrm{n}=$ 157) for $750 \mu \mathrm{M}, 78.38 \pm 5.10 \%(\mathrm{n}=145)$ for $1 \mathrm{mM}, 84.05$ $\pm 2.29 \%(\mathrm{n}=131)$ for $5 \mathrm{mM}$.

\section{Exposure to BPA disturbs the cell cycle of porcine oocyte maturation}

Since BPA treatment caused the failure of oocyte maturation, we extended the culture time to $60 \mathrm{~h}$ to check the cell cycle progression. As shown in Figure 2A, we observed the polar body of oocytes for $60 \mathrm{~h}$ with BPA culture. Most oocytes had extruded small polar bodies and were arrested at the MII stage in the control group, however most oocytes failed to extrude a polar body with $250 \mu \mathrm{M}$ BPA treatment. As shown in Figure 2B, COCs were treated with BPA for $44 \mathrm{~h}$, most control oocytes were arrested at meiosis II (MII), whereas most of BPA treated oocytes were arrested at Metaphase I (MI) and anaphase/ telophase I (ATI). The proportion of the germinal vesicle breakdown (GVBD) stage oocytes with BPA treatment $(3.57 \pm 1.00 \%, \mathrm{n}=151)$ was not significantly different from that in the control group $(6.52 \pm 4.53 \%, n=114)(\mathrm{P}>$ $0.1)$. In addition, the rates of MI and ATI stage oocytes were significantly increased $(40.18 \pm 2.26 \%$ for MI and $19.27 \pm 1.44 \%$ for ATI) compared with controls $(22.72$ $\pm 2.78 \%$ for $\mathrm{MI}$ and $0.57 \pm 0.57 \%$ for ATI; $<<0.01$ ), and the rates of MII stage oocytes were significantly decreased $(36.98 \pm 2.36 \%)$ compared with controls $(70.19 \pm 6.86 \%$, $\mathrm{p}<0.05)$. We also examined the proportion of oocytes stage for $60 \mathrm{~h}$ culture, and the result was similar to the above observation. The rates of MI stage were significantly increased to $36.57 \pm 2.11 \%$ when compared with that in controls $(15.34 \pm 2.01 \%)(\mathrm{p}<0.01)$, and similar results was shown at ATI stage $(17.17 \pm 6.94 \%, \mathrm{n}=144$ vs 0 , $\mathrm{n}=174)$; but the rates of MII were significantly reduced $(40.41 \pm 4.36 \%)$ compared with controls $(83.54 \pm 2.57 \%)$ $(\mathrm{p}<0.01)$. These results showed that BPA exposure blocked the cell cycle progression of porcine oocyte maturation. 


\section{BPA affects porcine oocyte cytoskeletal dynamics}

To investigate why the oocytes with BPA treatment reduced maturation competence, We firstly examined the morphologies of meiotic spindles of metaphase I (MI) oocytes after BPA treatment. The majority of control oocytes that were arrested at MI stage had normal spindle morphologies and well-aligned chromosomes. However, the majority of oocytes with BPA treatment had disrupted spindle morphologies and misaligned chromosomes. Spindles showed multiple poles, no poles, or disrupted poles, as shown in Figure 3A. The abnormal rates of oocytes with BPA treatment were significantly increased $(29.61 \pm 4.17 \%, \mathrm{n}=110)$ compared to controls $(6.35 \pm$ $0.14 \%, \mathrm{n}=96, \mathrm{p}<0.05$ ), as shown in Figure 3B.

Secondly, we assessed the localization of actin filaments. As shown in Figure 3C, compared with the controls for metaphase I (MI) oocytes after BPA exposure, actin fluorescence intensity on the membrane was significantly reduced $(66.55 \pm 4.81 \%, \mathrm{n}=12$, vs. $100 \% ; \mathrm{p}<0.05)$. Similarly, the actin fluorescence intensity in the cytoplasm was significantly lower $(70.06 \pm 6.26 \%, \mathrm{n}=12$, vs. $100 \%$; $\mathrm{p}<0.05$ ) than that in control MI oocytes (Figure 3D).
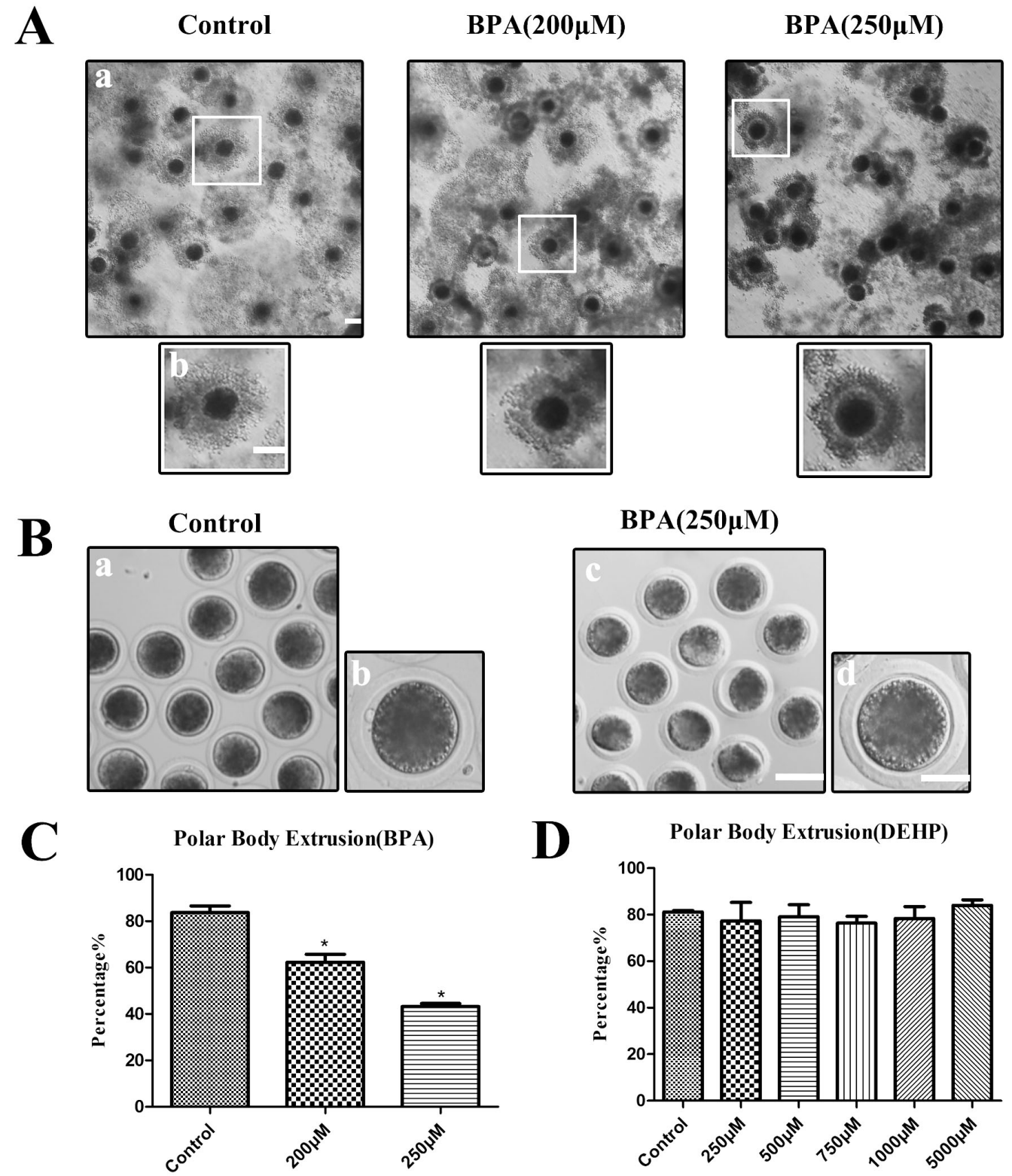

Figure 1: BPA treatment results in the failure of polar body extrusion in porcine oocytes but not DEHP in vitro. A. Granule cell diffusion of COCs were weakened after BPA treatment. a, Bar $=100 \mu \mathrm{m} ; \mathrm{b}, \mathrm{Bar}=50 \mu \mathrm{m}$. B. Oocytes failed to extrude polar bodies after BPA treatment. a, c Bar $=50 \mu \mathrm{m} ; \mathrm{b}, \mathrm{d}$ Bar $=20 \mu \mathrm{m}$. C. The polar body extrusion rate was significantly reduced after BPA treatment. Asterisks denote significantly different $(\mathrm{P}<0.01)$. *significantly different $(\mathrm{P}<0.01)$. D. The polar body extrusion rate no significantly variation after DEHP treatment $(\mathrm{P}>0.05)$. 
To further confirm the effects of BPA on meiotic spindle, we examined the typical spindle regulator MAPK. The oocytes were harvested at MI stage, and then the localization and expression level of p-MAPK were assessed by Western blot. We found that the expression of p-MAPK was decreased after BPA exposure (Figure 3E). For BPA treatment, the level of p-MAPK was significantly lower $(56.46 \pm 10.6 \%$ vs. $100 \% ; \mathrm{p}<0.05)$ compared to controls (Figure 3F).

\section{BPA treatment results in epigenetic alterations in porcine oocytes}

Epigenetic modifications were examined after BPA exposure. Firstly, we harvested the oocytes at the stage of GV and cultured for $26 \mathrm{~h}$ to the MI stage, then the oocytes were observed by a confocal microscope after immunofluorescence staining. The levels of histone lysine methylation were examined, and we found that H3K4me2 was co-localized with DNA (Figure 4A); however, compared with controls, the levels of $\mathrm{H} 3 \mathrm{~K} 4 \mathrm{me} 2$ methylation with BPA treated was significantly reduced $(0.77 \pm 0.39$ vs. $1.0 ; p<0.05 ;$ Figure $4 \mathrm{~B})$. The oocytes were harvested at the stage of MI for $26 \mathrm{~h}$, the levels of mRNA expression for DNA methyl-transferases (Ash2l, Suv39h2, Eed, Ezh2, Suz12) were examined by quantitative PCR analysis (Figure 4C). After BPA exposure, the relative mRNA expression of $A s h 2 l$, Eed and Ezh2, significantly increased compared to the control oocytes $(1.66 \pm 0.16$, $1.44 \pm 0.09$ and $1.71 \pm 0.17$ vs. 1.0$)(\mathrm{P}<0.05)$. The relative mRNA expression of Suz12 and Suv39h2 were not significantly increased $(1.67 \pm 0.39$ and $1.19 \pm 0.37$ vs. 1.0) when compared with that in controls $(\mathrm{P}>0.1)$.

Secondly, the levels of $5 \mathrm{mC}$ were examined, as shown in Figure 4D, we observed that $5 \mathrm{mC}$ was also colocalized with DNA. Compared with the controls for which the relative intensity of $5 \mathrm{mC}$ expression was significantly decreased $(54.64 \pm 7.70 \%$ vs.100\%) $(\mathrm{P}<0.05)$ after BPA treatment (Figure 4E). Subsequently, the levels of mRNA expression for DNA methyl-transferases (Dnmt3a, $D n m t 3 b$ ) were assessed by quantitative PCR analysis. The relative mRNA expression of Dnmt3b was significantly decreased $(0.67 \pm 0.09$ vs. 1.0$)$ when compared with that in controls $(\mathrm{P}<0.05)$. The relative expression of Dnmt3a mRNA levels had no significant variation when compared with the controls $(0.98 \pm 0.22$ vs. 1.0$)(\mathrm{P}>0.1)$ (Figure $4 \mathrm{~F})$.

\section{BPA treatment increases ROS generation in porcine oocytes}

Next, the levels of ROS were examined after $26 \mathrm{~h}$ culture. As shown in Figure 5A, compared with controls the fluorescent intensity of ROS was increased
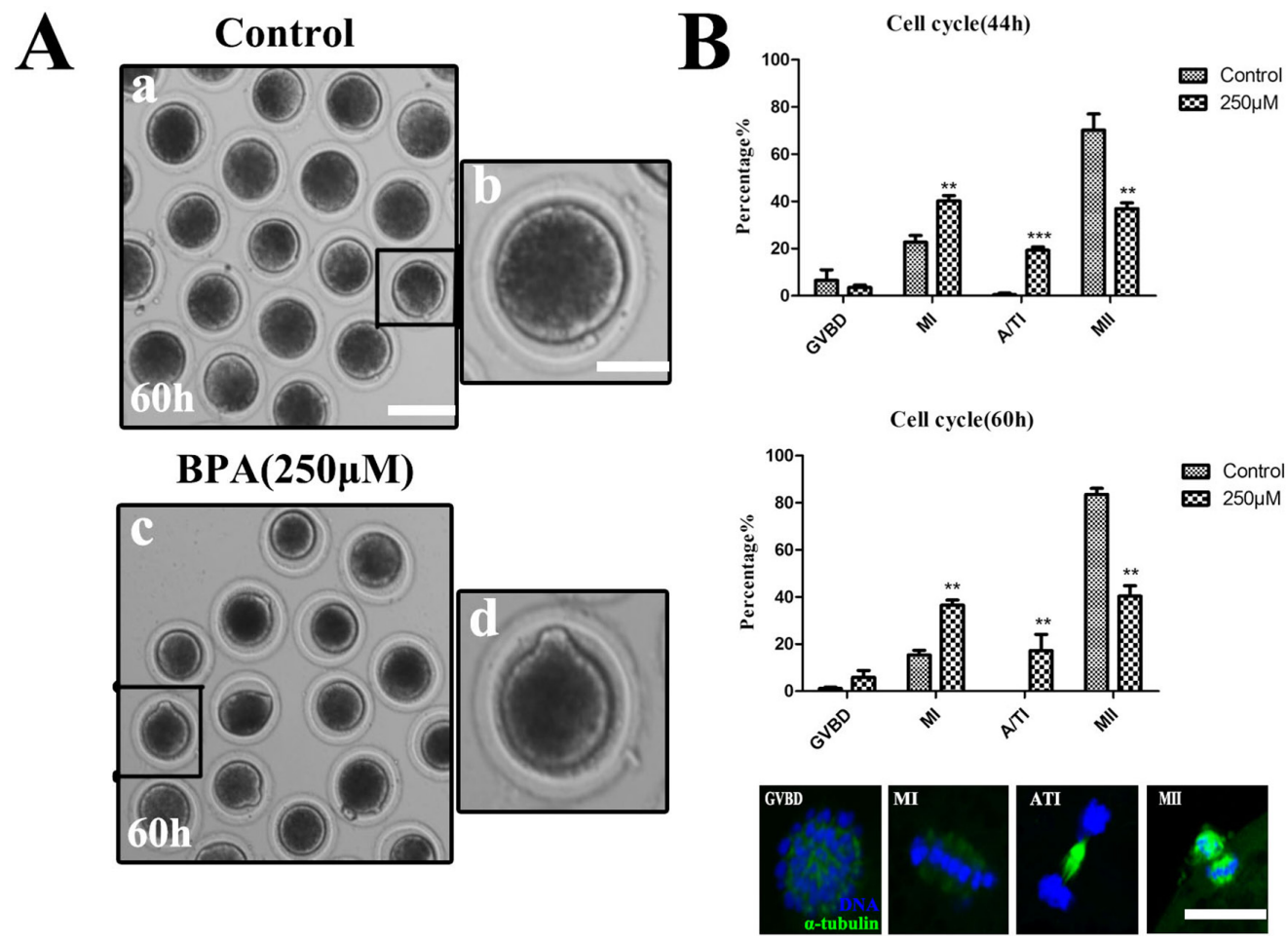

Figure 2: Exposure to BPA disturbs the cell cycle of porcine oocyte maturation. A. Oocytes failed to extrude polar bodies after exposure to BPA for $60 \mathrm{~h}$ culture. a, c Bar $=80 \mu \mathrm{m}$; b, d Bar $=20 \mu \mathrm{m}$. B. The rates of different stages for $44 \mathrm{~h}$ and $60 \mathrm{~h}$ cultured. (For $44 \mathrm{~h}$, Control $n=114$, Treatment $n=151$ ) (For $60 h$, Control $n=144$, Treatment $n=174$ ), $* P<0.05$, the typical stage of porcine oocytes were displayed by the fluorescence images. Green, spindle; blue, chromatin. Bar $=5 \mu \mathrm{m}$. 
in the treatment group by immunofluorescent staining (Figure 5A). The relative fluorescence intensity of ROS was significantly increased (3.46 \pm 0.82 vs.1.0 for control) $(\mathrm{P}<0.05)$ after BPA treatment for $26 \mathrm{~h}$ culture (Figure 5B). The levels of mRNA expression for oxidative stress-related genes (CAT, SOD1, SOD2, Prdx2, PrdxO) were examined by quantitative PCR analysis (Figure $5 \mathrm{C})$. Only the relative mRNA expression of SOD1 was significantly increased when compared with the controls $(1.36 \pm 0.13$ vs. 1.0$)(\mathrm{P}<0.05)$. And the relative mRNA expression of CAT, SOD2, Prdx2 and Prdx6 had no significantly variation: $1.28 \pm 0.15,1.65 \pm 0.36,2.29 \pm$ 1.08 and $1.37 \pm 0.21$ vs. 1.0 for controls $(\mathrm{P}>0.1)$.

\section{BPA treatment results in early apoptosis and autophagy in porcine oocytes}

Apoptosis and autophagy were examined at the stage of MI after $26 \mathrm{~h}$ culture. Firstly, the green fluorescent signals were stained by Annexin- $\mathrm{V}$ staining. In control oocytes, Annexin-V staining signals showed at the zona pellucida and were non-apoptotic, but the clear green signals were found in the membrane and zona pellucida after BPA treatment (Figure 6A). As shown in Figure 6B, the green fluorescent signals were significantly increased after BPA treatment $(64.31 \pm 2.46 \%, \mathrm{n}=10)$ compared with controls $(35.69 \pm 2.46 \%, \mathrm{n}=10)(\mathrm{P}<0.05)$. The levels of mRNA expression for apoptosis-related genes ( $B A K, B c l-x l$ ) were examined by quantitative PCR analysis (Figure $6 \mathrm{C}$ ). The relative mRNA expression of $B c l-x l$ was significantly decreased when compared to the control oocytes $(0.66 \pm 0.12$ vs. 1.0$)(\mathrm{P}<0.05)$, while the relative expression of $B A K \mathrm{mRNA}$ levels had no apparent variation when compared with the controls $(1.05 \pm 0.12$ vs. 1.0$)$ $(\mathrm{P}>0.1)$.

Next, as shown in Figure 6D, the status of autophagy were stained by the expression of microtubuleassociated protein light chain 3 (LC3), and we found that more dots appeared in the treated oocytes than those in the control oocytes after $26 \mathrm{~h}$ culture. Furthermore, the levels of LC3 protein levels were significantly increased after BPA treatment by Western blot (Figure 6E). For control oocytes, the relative intensity of LC3 expression was significantly increased $(2.08 \pm 0.26$ vs. 1.0$)(\mathrm{P}<0.05)$
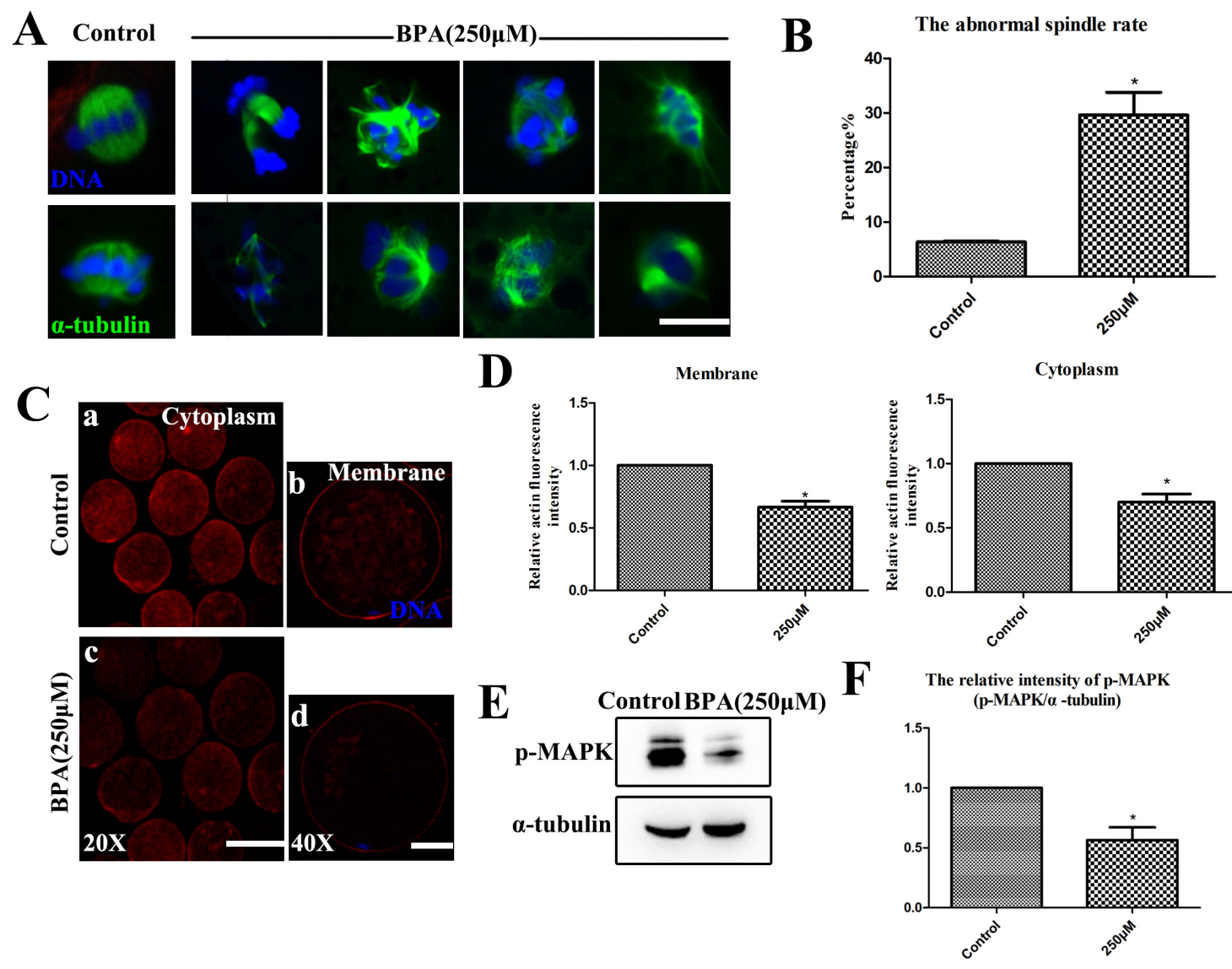

Figure 3: BPA results in oocyte cytoskeletal abnormalities during porcine oocyte maturation. A. The spindle formation was disrupted after BPA treatment. Bar $=5 \mu \mathrm{m} \mathrm{B}$. The rates of abnormal spindle formation was significantly increased $(\mathbf{P}<0.05)$. C and $\mathbf{D}$. The expression of actin signals at the membrane and in the cytoplasm of MI oocytes were significantly reduced when compared with controls $(\mathrm{P}<0.05)$. a , c Bar $=50 \mu \mathrm{m} ; \mathrm{b}, \mathrm{d}$ Bar $=20 \mu \mathrm{m}$. E. The localization and expression level of p-MAPK were assessed by Western blot. F. The relative intensity of p-MAPK protein expression ( $\mathrm{p}-\mathrm{MAPK} / \mathrm{a}-\mathrm{tubulin})$ was significantly reduced $(\mathrm{P}<0.05)$. Blue, chromatin; Green, tubulin; Red, actin. $* \mathrm{P}<0.05$. 
after BPA treatment (Figure 6F). The levels of mRNA expression for autophagy-related genes ( $m T O R, L C 3$, LAMP2, Beclin1, ATG3, ATG5, ATG7) were examined by quantitative PCR analysis (Figure $6 \mathrm{G}$ ). The relative mRNA expression of LC3, ATG3 and ATG7 were significantly increased when compared to controls $(1.26$ $\pm 0.03,1.63 \pm 0.19$ and $2.15 \pm 0.22$ vs. 1.0$)(\mathrm{P}<0.05)$. But the relative mRNA expression of $m T O R$ and Beclin 1 were significantly decreased when compared to the controls $(0.51 \pm 0.09$ and $0.58 \pm 0.03$ vs. 1.0$)(\mathrm{P}<0.05)$. The results of $L A M P 2$ and $A T G 5$ relative mRNA expression had no significant variation: $1.55 \pm 0.64$ for $L A M P 2$ and $1.7955 \pm$ 0.73 for $A T G 5$ vs.1.0 for controls ( $\mathrm{P}>0.1$ ).

\section{DISCUSSION}

BPA is an endocrine disruptor which could impair human reproductive capacity, and DEHP is an estrogenic chemical that is ubiquitously used in plastics and common consumer products. In this study, our results indicated that exposure to BPA caused failure of polar body extrusion on porcine oocytes in vitro, but not DEHP. This may be because that when DEHP enters the gastro-intestinal tract, it is rapidly metabolized to mono(2-ethylhexyl) phthalate (MEHP) and 2-ethylhexanol via pancreatic lipases, while the MEHP has toxic effects instead of DEHP. Subsequently, the toxicity of BPA was demonstrated and its potential mechanisms were explored, including cytoskeletons, epigenetic modification, oxidative stress, autophagy and apoptosis.

Previous study showed that the numbers of porcine COCs which reached complete expansion were significantly reduced after exposure of FSH-stimulated OCC to BPA [30]. BPA exposure suppressed polar body release in mice [31], and exposure to BPA during in vitro oocyte maturation could decrease oocyte quality in Bos taurus [32]. In addition, there was a dose-response
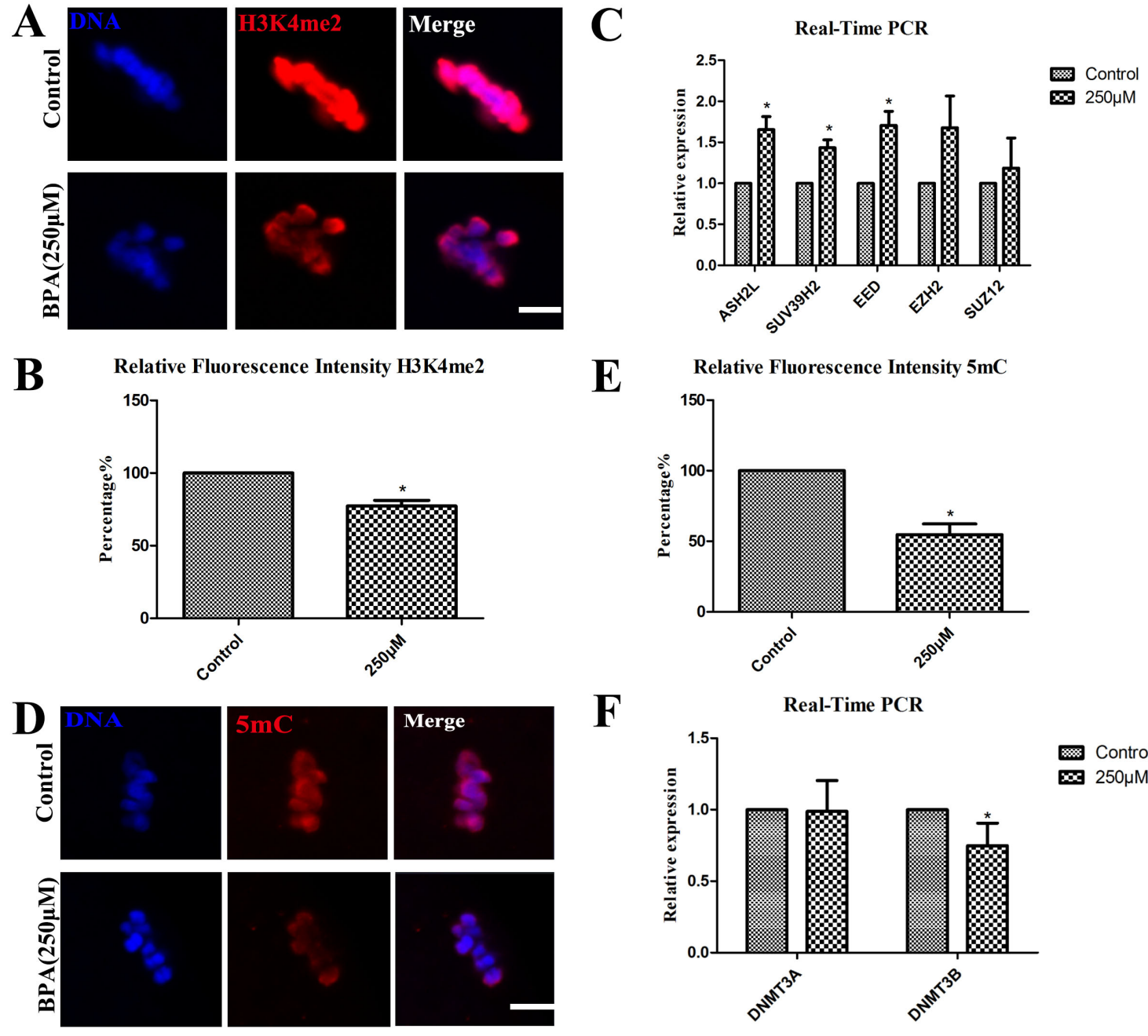

E

Relative Fluorescence Intensity $5 \mathrm{mC}$
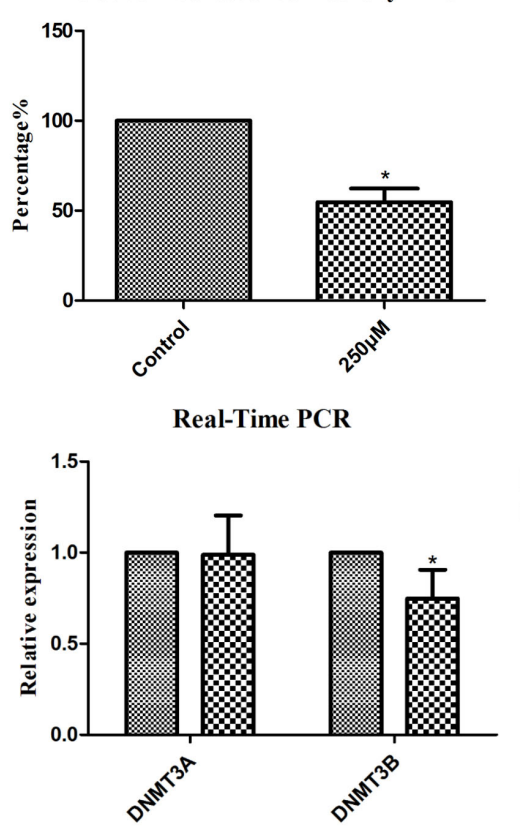

Figure 4: BPA treatment results in epigenetic alterations in porcine oocytes. A and B. The expression of Di-methyl-Histone H3 (Lys4) (H3K4me2) was decreased after BPA treatment by immunofluorescent staining, the fluorescence intensity analysis was significantly decreased $(\mathrm{P}<0.05)$. C. ASH $2 L, S U V 39 H 2$ and EED mRNA levels were significantly increased after BPA treatment. D and E. The expression of 5 methyl cytosine $(5 \mathrm{mC})$ was decreased after exposure to BPA, the fluorescence intensity analysis was significantly decreased $(\mathrm{P}<0.05)$. F. DNMT3B mRNA levels were significantly decreased after BPA treatment. $* \mathrm{P}<0.05$. Bar $=5 \mu \mathrm{m}$. 
association of BPA exposure as for altering the maturation of human oocytes in vitro [22]. While our results showed that the expansion of cumulus cells was weakened after BPA treatment, and the rates of polar body extrusion were reduced with BPA treatment in porcine oocytes. However, the polar body extrusion rates have no significant variation after DEHP exposure. Our results indicated that similar with other models, BPA had directly toxic effects on porcine oocytes in vitro, but not DEHP. In mice, oocytes exposed to high doses of BPA experienced a cell cycle delay, and managed to progress to MII when the culture period was prolonged [31]. And short exposure to BPA during the final stages of oocyte maturation is associated with cell cycle delays and spindle abnormalities $[17,22$, 33]. However, our study showed that BPA blocked the cell cycle progression of porcine oocyte maturation even after culturing $60 \mathrm{~h}$. This indicated that porcine oocytes were more sensitive to the BPA exposure.

Actin microfilament and spindle formation are crucial for oocyte meiotic maturation and fertilization. BPA exhibited a significantly higher incidence of meiotic arrest and spindle abnormalities after exposed denuded mouse oocytes in vitro [17]. Similar to their findings, our results showed that BPA exposure disrupted spindle formation in porcine oocytes, which also was confirmed by the decreased expression of phosphorylation of MAPK, a regulator which regulated spindle formation [34]. Actin filament is critical for cytokinesis and polar body extrusion, and we also observed reduced the expression of membrane and cytoskeletal actin after BPA treatment. This provided a reason for the failure of polar body extrusion from subcellular structure level.
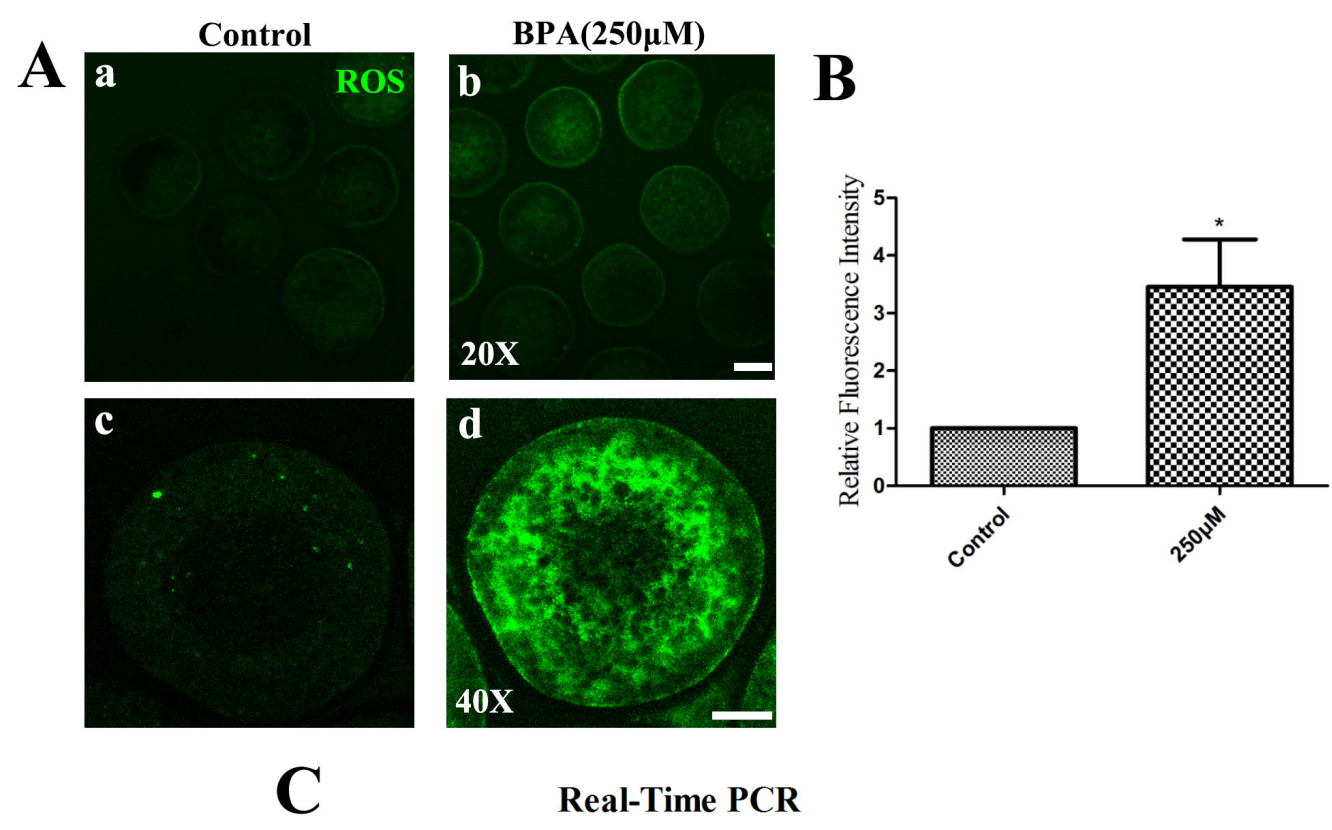

\section{Real-Time PCR}

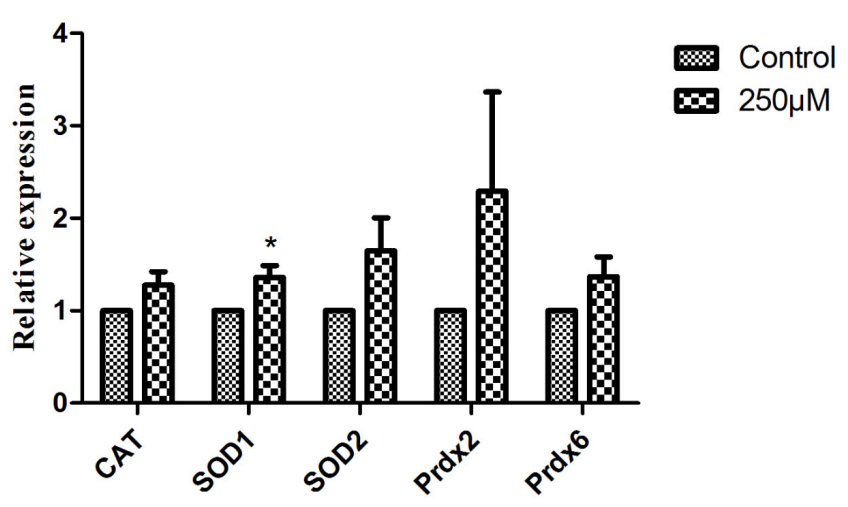

Figure 5: BPA effects on porcine oocyte early apoptosis and autophagy. A. BPA induced early-stage apoptosis. In control oocytes, it was only show fluorescence on the zona, whereas oocytes exhibited fluorescence on the zona and oocyte membrane after BPA treatment. a, c Bar $=50 \mu \mathrm{m} ; \mathrm{b}, \mathrm{d}$ Bar $=20 \mu \mathrm{m}$. B. The early apoptosis rate was significantly increased $(\mathrm{P}<0.05)$. C. $B c l-x l \mathrm{mRNA}$ levels were significantly decreased after BPA treatment. * $\mathrm{P}<0.05$. Bar $=20 \mu \mathrm{m}$. D. Oocytes were stained by immunofluorescence staining after BPA treatment, there are more LC3 puncta than control oocytes. Bar $=20 \mu \mathrm{m}$. $\mathbf{E}$ and $\mathbf{F}$. The localization and expression level of LC3 were assessed by Western blot, therelative intensity of LC3 protein expression (LC3/ $\alpha$-tubulin) was significantly increased (P $<0.01)$. G. LC3, $A T G 3$ and $A T G 7$ mRNA levels were significantly increased after BPA treatment, but mTOR and Beclin1 mRNA levels were significantly decreased after BPA exposure. $* \mathrm{P}<0.05$. 
The epigenetic effects include histone modification, DNA methylation and the formation of histone variants [35]. Previous work showed that BPA might contribute to chromosome congressional failures, and altered gene expression that might affect the health of the offspring [36]. In our results, the decreased level of $\mathrm{H} 3 \mathrm{~K} 4 \mathrm{me} 2$ indicated that BPA had altered oocyte histone modifications. We also found some epigenetic genes increased after BPA treatment. Because fully grown oocytes are transcriptionally inactive, then the "increases" in mRNA observed might only reflect a failure to degrade the mRNAs. DNA methylation studies shown that BPA exposure significantly changed the methylation levels of differentially methylated regions (DMRs) including the Snrpn imprinting control region (ICR) and Igf2 DMR1 [37]. Thus, the reduced $5 \mathrm{mC}$ expression in our results indicated that BPA had disturbed oocyte DNA methylation status and might further inhibited meiotic progression of oocytes.

Apoptosis also plays a major role in all the stages of oogenesis and even after ovulation [24, 38, 39]. And a previous study showed that implication of autophagy against BPA-mediated neurodegeneration through involvement of AMPK and mTOR pathways [40]. Our results showed that BPA induced early apoptosis and autophagy in porcine oocytes which was confirmed by the levels of LC3 and autophagy-related genes mRNA expression. To further explore this, we also examined the oxidative stress levels. And we found that the porcine oocytes suffered oxidative stress after BPA treatment, showing with increased levels of ROS and oxidative stress-related gene mRNA expression. This result is similar with the previous study which showed that BPA induced oxidative toxicity and carcinogenic effects with a specific dose level [41]. Reactive oxygen species (ROS) play a role in the physiological processes of meiotic resumption as key signal molecules, and including the induction of cell apoptosis and senescence [42]. Therefore, our results indicated that BPA exposure induced ROS, which may further induce apoptosis/autophagy.

In summary, our results showed that the effects of BPA treatment on oocyte cytoskeletons, epigenetic modification, oxidative stress, autophagy and apoptosis of porcine oocytes, which further provided the evidence for the toxic effects of BPA on reproductive systems.
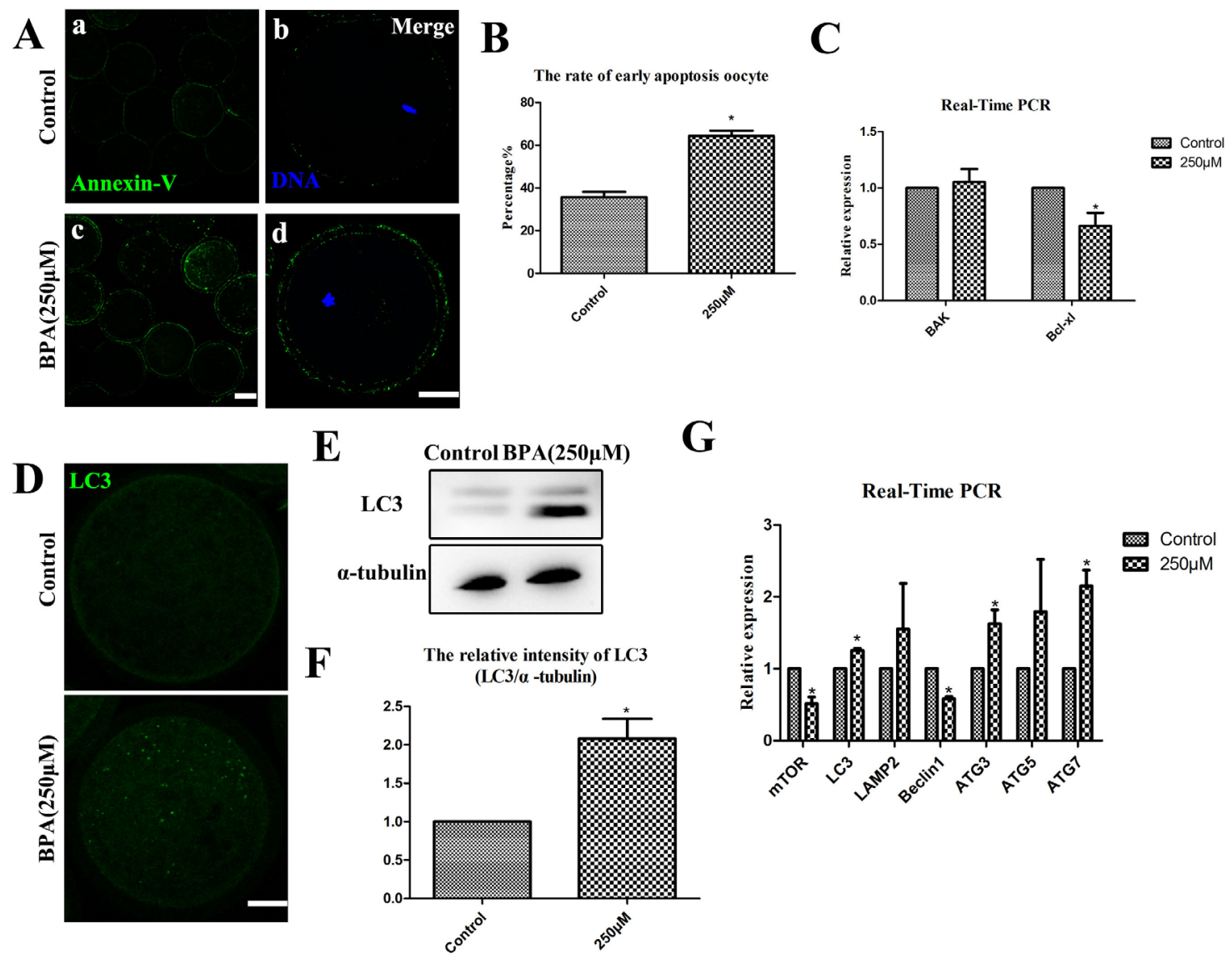

Figure 6: BPA effects on ROS generation after BPA treatment. A. ROS generation was obviously increased after BPA treatment. $\mathrm{a}, \mathrm{b}$ Bar $=50 \mu \mathrm{m} ; \mathrm{c}, \mathrm{d}$ Bar $=20 \mu \mathrm{m}$. B. The relative fluorescence intensity of ROS was significantly increased $(\mathrm{P}<0.05)$. C. The oxidative stress-related genes were examined. SOD1 mRNA levels were significantly increased after BPA treatment $(\mathrm{P}<0.05)$, while there is no change for the mRNA level of CAT, SOD2, Prdx2 and Prdx6. 


\section{MATERIALS AND METHODS}

\section{Antibodies and chemicals}

BPA and DEHP were purchased from J\&K Chemical Ltd. (Shanghai, China). Mouse monoclonal anti- $\alpha$-tubulinFITC, phalloidin-TRITC (actin) and Hoechst 33342 were from Sigma (St. Louis, MO, USA). Rabbit polyclonal anti-H3K4me2 antibody, rabbit monoclonal anti-p-MAPK antibody and rabbit polyclonal LC3 antibody were from Cell Signaling Technology (Devers, MA, USA). Mouse monoclonal anti-5-methyl cytosine $(5 \mathrm{mC})$ antibody was purchased from Abcam (Cambridge, UK). Annexin V-FITC/EGFP Apoptosis Detection Kits were purchased from Vazyme Biotech Co., Ltd. (Nanjing, China). Alexa Fluor 488 goat anti-rabbit, 594 goat anti-mouse antibodies were from Invitrogen (Carlsbad, CA, USA).

\section{BPA and DEHP treatment}

BPA and DEHP were dissolved in DMSO respectively, and then BPA was diluted into a final concentration of $200 \mu \mathrm{M}$ and $250 \mu \mathrm{M}$ with maturation medium; meanwhile, DEHP was diluted with maturation medium to final concentrations of $250 \mu \mathrm{M}, 500 \mu \mathrm{M}$, $750 \mu \mathrm{M}, 1 \mathrm{mM}$ and $5 \mathrm{mM}$, both of them with the final concentration of the solvent not more than $1 \%$ of the culture medium.

\section{Immunofluorescence staining}

Oocytes were fixed with $4 \%(\mathrm{w} / \mathrm{v})$ paraformaldehyde in phosphate buffered saline (PBS) at room temperature for $30 \mathrm{~min}$ which for immunofluorescent staining. Then they were transferred to a membrane permeabilization solution (1\% Triton X-100 in PBS) for $8-12 \mathrm{~h}$ at room temperature. In blocking buffer $(1 \%$ bovine serum albumin [BSA] in PBS) for $1 \mathrm{~h}$, oocytes were subsequently incubated overnight at $4^{\circ} \mathrm{C}$ or $4 \mathrm{~h}$ at room temperature with different primary antibodies (H3K4me2, 1:1000; LC3, 1:100). After three washes (2 min each) in washing buffer containing $0.1 \%$ Tween 20 and $0.01 \%$ Triton X-100 in PBS, oocytes were stained for $1 \mathrm{~h}$ with Alexa Fluor secondary antibodies at room temperature. For spindle and actin examination, oocytes were incubated with anti- $\alpha$ tubulin-FITC and Phalloidin-TRITC staining for $1 \mathrm{~h}$, then samples were counterstained with Hoechst $33342(10 \mu \mathrm{g} /$ $\mathrm{ml}$ in PBS) for $10 \mathrm{~min}$ and then followed by three washes in washing buffer.

Detecting the fluorescence signal of $5 \mathrm{mC}$, the zona pellucid of oocytes were removed $(0.05 \%$ pronase in PBS). These oocytes were denatured with $2 \mathrm{~N} \mathrm{HCl}$ at room temperature for $30 \mathrm{~min}$ and neutralized with 100 $\mathrm{mM}$ Tris- $\mathrm{HCl}, \mathrm{pH} 8.5$ for $10 \mathrm{~min}$, then incubated in PBS containing $0.05 \%$ Tween- 20 at $4^{\circ} \mathrm{C}$ overnight or at room temperature with for $1 \mathrm{~h}$ followed by washing five times in phosphate-buffered saline (PBS) that contained 1\% BSA (PB1), then these oocytes were incubated overnight at $4{ }^{\circ} \mathrm{C}$ with a mouse anti-5-mC antibody (1:500).

Specimens were mounted on glass slides and examined with a confocal laser-scanning microscope (Zeiss LSM 700 META, Germany). In each experiment was repeated at least three times and at least 10 oocytes were examined for each group.

\section{Oocyte treatment and in vitro maturation}

Porcine ovaries were collected from prepubertal gilts at a local slaughterhouse and transported to our laboratory in physiological saline $(0.9 \% \mathrm{NaCl})$ containing $500 \mathrm{IU} /$ $\mathrm{ml}$ of both penicillin and streptomycin at $30-35^{\circ} \mathrm{C}$ within $2 \mathrm{~h}$ kept in a thermos bottle after slaughter. Subsequently ovaries were washed twice with sterile phosphate-buffered saline (PBS), then COCs (cumulus oocyte complexes) were aspirated from antral follicles (2-5 $\mathrm{mm}$ in diameter) using a 20 -gauge needle attached to a $10-\mathrm{ml}$ disposable syringe. After washing three times with maturation medium, the COCs with intact and compact cumulus were separated from the cellular debris. The medium used for maturation culture was improved TCM-199 supplemented with $75 \mu \mathrm{g} / \mathrm{ml}$ of penicillin, $50 \mu \mathrm{g} / \mathrm{ml}$ of streptomycin, 0.5 $\mu \mathrm{g} / \mathrm{ml}$ of LH, $0.5 \mu \mathrm{g} / \mathrm{ml}$ of FSH, $10 \mathrm{ng} / \mathrm{ml}$ of epidermal growth factor (mouse EGF; Sigma) and $0.57 \mathrm{mM}$ cysteine (Sigma). To prepare mature oocytes in vitro, a group of 80 COCs were transferred to $500 \mu \mathrm{l}$ of maturation medium, then medium were subsequently covered with $200 \mu \mathrm{l}$ paraffin oil in a four-well dish at $38.5^{\circ} \mathrm{C}$ in a humidified atmosphere of 5\% $\mathrm{CO}^{2}$ (Nunc, Roskilde, Denmark).

\section{Annexin-V staining of oocytes}

According to the manufacturer's instructions (Beyotime Institute of Biotechnology Hangzhou, China), oocytes were stained with an Annexin-V staining kit. After washing twice in PBS, then viable oocytes were stained for $10 \mathrm{~min}$ in the dark with $100 \mathrm{ml}$ of binding buffer containing $5 \mathrm{ml}$ of Annexin-V-FITC. Fluorescent signals were measured by a confocal microscope (Zeiss LSM 700 META).

\section{Determination of ROS generation}

To determine the levels of intracellular ROS production, cumulus-denuded oocytes were incubated with the oxidation-sensitive fluorescent probe [dichlorofluorescein $(\mathrm{DCFH})$ ] for $30 \mathrm{~min}$ at $37^{\circ} \mathrm{C}$ in D-PBS that contained $10 \mu \mathrm{M}$ DCFH diacetate (DCFHDA) (Beyotime Institute of Biotechnology, China). Then oocytes were washed three times in D-PBS containing $0.1 \%$ BSA and then placed on glass slides. The measurement of the intensity of florescence in each oocytes was by a Zeiss LSM 700 META confocal system with the same scanning settings were used for each sample. 


\section{Protein extraction and western blot analysis}

A total of 100 porcine oocytes were collected at the MI stage, and then lysed in Laemmli sample buffer (SDS sample buffer with 2-mercaptoethanol), oocytes were subsequently boiled at $100^{\circ} \mathrm{C}$ for $10 \mathrm{~min}$ and immediately transferred to fridge to frozen at $-20^{\circ} \mathrm{C}$ until use. Proteins were separated by SDS polyacrylamide gel electrophoresis (PAGE) using $8 \%$ gels and then transferred onto a polyvinylidene fluoride membrane (Millipore, Billerica, MA). Membranes were blocked with Trisbuffered saline (TBS) containing $0.1 \%$ (w/w) Tween 20 (TBST) and 5\% (w/v) nonfat dry milk powder for $1.5 \mathrm{~h}$ at room temperature, and then incubated with a rabbit monoclonal antibody $(1: 2000$; for $\mathrm{p}-\mathrm{MAPK}$ and LC3, incubation buffer was 5\% BSA in PBST) overnight at $4^{\circ} \mathrm{C}$. After washing three times in PBST (10 min each), membranes were incubated for $1 \mathrm{~h}$ with secondary anti-rabbit HRP-conjugated antibodies (1:2000) in 5\% nonfat dry milk in TBST. Finally, the membrane was exposed to enhanced chemiluminescence reagent (EMD Millipore, Billerica, MA).

\section{Real-time quantitative PCR}

30 oocytes were used to extract total RNA with a Dynabead mRNA DIRECT kit (Invitrogen Dynal, Oslo, Norway). First strand cDNA was synthesized using a cDNA synthesis kit (Takara) using Oligo(dT) 12-18 primers (Invitrogen) according to the manufacturer's instructions (Invitrogen). These cDNAs were diluted 10 times and stored at $-20^{\circ} \mathrm{C}$ until analysis. Quantitative realtime PCR (qRT-PCR) was conducted with a fast real-time PCR system (ABI Step One Plus). GAPDH was used as a control gene and triplicate samples were assessed for each gene of interest. Relative expression levels were determined by the $2^{-\Delta \Delta \mathrm{Ct}}$ method.

\section{Statistical analysis}

For each treatment, at least three biological replicates were done and the results were expressed as means \pm SEMs. The variance (ANOVA) analysis were used for Statistical comparisons and Duncan's multiple comparisons test were used in differences between BPA treatment groups. The value of $\mathrm{p}<0.05$ was significant.

\section{FUNDING}

This study was supported by the National Basic Research Program of China (2014CB138503); and the Biogreen 21 Program (PJ011126), RDA, Republic of Korea.

\section{Authors' roles}

The main experimental conception and design: T.W., S.C.S.; Performed the experiments: T.W.; Analyzed the data and contributed reagents: T.W., J.H, X.D. X.S.C, N.H.K and S.C.S.; Writing the manuscript: T.W., B. X., S.C.S. All the authors approved the final version.

\section{CONFLICTS OF INTEREST}

The authors have no conflicts of interest to disclose.

\section{REFERENCES}

1. Lyche JL, Gutleb AC, Bergman A, Eriksen GS, Murk AJ, Ropstad E, Saunders M and Skaare JU. Reproductive and developmental toxicity of phthalates. Journal of Toxicology and Environmental Health Part B Crit Rev. 2009; 12:225-249.

2. Mathieu-Denoncourt J, Wallace SJ, de Solla SR and Langlois VS. Plasticizer endocrine disruption: Highlighting developmental and reproductive effects in mammals and non-mammalian aquatic species. General and Comparative Endocrinology. 2015; 219:74-88.

3. Zhang XF, Zhang LJ, Li L, Feng YN, Chen B, Ma JM, Huynh E, Shi QH, De Felici M and Shen W. Diethylhexyl phthalate exposure impairs follicular development and affects oocyte maturation in the mouse. Environmental and Molecular Mutagenesis. 2013; 54:354-361.

4. Kavlock R, Barr D, Boekelheide K, Breslin W, Breysse P, Chapin R, Gaido K, Hodgson E, Marcus M, Shea K and Williams P. NTP-CERHR Expert Panel Update on the Reproductive and Developmental Toxicity of di(2ethylhexyl) phthalate. Reproductive Toxicology. 2006; 22:291-399.

5. Latini G. Monitoring phthalate exposure in humans. Clinica Chimica Acta. 2005; 361:20-29.

6. Ambruosi B, Uranio MF, Sardanelli AM, Pocar P, Martino NA, Paternoster MS, Amati F and Dell'Aquila ME. In vitro acute exposure to DEHP affects oocyte meiotic maturation, energy and oxidative stress parameters in a large animal model. PloS One. 2011; 6:e27452.

7. Steinmetz R, Brown NG, Allen DL, Bigsby RM and Ben-Jonathan N. The environmental estrogen bisphenol A stimulates prolactin release in vitro and in vivo. Endocrinology. 1997; 138:1780-1786.

8. Zoeller RT. Environmental chemicals impacting the thyroid: targets and consequences. Thyroid. 2007; 17:811-817.

9. Wetherill YB, Akingbemi BT, Kanno J, McLachlan JA, Nadal A, Sonnenschein C, Watson CS, Zoeller RT and Belcher SM. In vitro molecular mechanisms of bisphenol A action. Reproductive Toxicology. 2007; 24:178-198.

10. Castro B, Sanchez P, Torres JM, Preda O, del Moral RG and Ortega E. Bisphenol A exposure during adulthood alters expression of aromatase and 5alpha-reductase isozymes in rat prostate. PloS One. 2013; 8:e55905.

11. Bonefeld-Jorgensen EC, Long M, Hofmeister MV and Vinggaard AM. Endocrine-disrupting potential of bisphenol A, bisphenol A dimethacrylate, 4-n-nonylphenol, and 4-n-octylphenol in vitro: new data and a brief review. Environmental Health Perspectives. 2007; 115 Suppl 1:69-76. 
12. Baker TG. A Quantitative and Cytological Study of Germ Cells in Human Ovaries. Proceedings of the Royal Society of London Series B, Biological Sciences. 1963; 158:417-433.

13. Knez J. Endocrine-disrupting chemicals and male reproductive health. Reproductive Biomedicine Online. 2013; 26:440-448.

14. Dobrzynska MM and Radzikowska J. Genotoxicity and reproductive toxicity of bisphenol A and X-ray/bisphenol A combination in male mice. Drug and Chemical Toxicology. 2013; 36:19-26.

15. Wright $\mathrm{C}$, Milne $\mathrm{S}$ and Leeson H. Sperm DNA damage caused by oxidative stress: modifiable clinical, lifestyle and nutritional factors in male infertility. Reproductive Biomedicine Online. 2014; 28:684-703.

16. Nagel SC, vom Saal FS, Thayer KA, Dhar MG, Boechler $\mathrm{M}$ and Welshons WV. Relative binding affinity-serum modified access (RBA-SMA) assay predicts the relative in vivo bioactivity of the xenoestrogens bisphenol A and octylphenol. Environmental Health Perspectives. 1997; 105:70-76.

17. Eichenlaub-Ritter U, Vogt E, Cukurcam S, Sun F, Pacchierotti F and Parry J. Exposure of mouse oocytes to bisphenol A causes meiotic arrest but not aneuploidy. Mutation Research. 2008; 651:82-92.

18. Lenie S, Cortvrindt R, Eichenlaub-Ritter U and Smitz J. Continuous exposure to bisphenol A during in vitro follicular development induces meiotic abnormalities. Mutation Research. 2008; 651:71-81.

19. Chao HH, Zhang XF, Chen B, Pan B, Zhang LJ, Li L, Sun $\mathrm{XF}$, Shi QH and Shen W. Bisphenol A exposure modifies methylation of imprinted genes in mouse oocytes via the estrogen receptor signaling pathway. Histochemistry and Cell Biology. 2012; 137:249-259.

20. Hunt PA, Koehler KE, Susiarjo M, Hodges CA, Ilagan A, Voigt RC, Thomas S, Thomas BF and Hassold TJ. Bisphenol a exposure causes meiotic aneuploidy in the female mouse. Current Biology. 2003; 13:546-553.

21. Muhlhauser A, Susiarjo M, Rubio C, Griswold J, Gorence G, Hassold T and Hunt PA. Bisphenol A effects on the growing mouse oocyte are influenced by diet. Biology of Reproduction. 2009; 80:1066-1071.

22. Machtinger R, Combelles CM, Missmer SA, Correia KF, Williams P, Hauser R and Racowsky C. Bisphenol-A and human oocyte maturation in vitro. Human Reproduction. 2013; 28:2735-2745.

23. Tiwari M, Prasad S, Tripathi A, Pandey AN, Ali I, Singh AK, Shrivastav TG and Chaube SK. Apoptosis in mammalian oocytes: a review. Apoptosis. 2015; 20:1019-1025.

24. Zhang HQ, Zhang XF, Zhang LJ, Chao HH, Pan B, Feng YM, Li L, Sun XF and Shen W. Fetal exposure to bisphenol A affects the primordial follicle formation by inhibiting the meiotic progression of oocytes. Molecular Biology Reports. 2012; 39:5651-5657.

25. Zhu CC, Zhang Y, Duan X, Han J and Sun SC. Toxic effects of HT-2 toxin on mouse oocytes and its possible mechanisms. Archives of Toxicology. 2015.

26. Xu YN, Shen XH, Lee SE, Kwon JS, Kim DJ, Heo YT, Cui XS and Kim NH. Autophagy influences maternal mRNA degradation and apoptosis in porcine parthenotes developing in vitro. The Journal of Reproduction and Development. 2012; 58:576-584.

27. Song BS, Kim JS, Kim YH, Sim BW, Yoon SB, Cha JJ, Choi SA, Yang HJ, Mun SE, Park YH, Jeong KJ, Huh JW Lee SR, et al. Induction of autophagy during in vitro maturation improves the nuclear and cytoplasmic maturation of porcine oocytes. Reproduction, Fertility, and Development. 2014; 26:974-981.

28. Tamura H, Takasaki A, Miwa I, Taniguchi K, Maekawa R, Asada H, Taketani T, Matsuoka A, Yamagata Y, Shimamura K, Morioka H, Ishikawa H, Reiter RJ, et al. Oxidative stress impairs oocyte quality and melatonin protects oocytes from free radical damage and improves fertilization rate. Journal of Pineal Research. 2008; 44:280-287.

29. Xin F, Jiang L, Liu X, Geng C, Wang W, Zhong L, Yang $\mathrm{G}$ and Chen M. Bisphenol A induces oxidative stressassociated DNA damage in INS-1 cells. Mutation Research Genetic Toxicology and Environmental Mutagenesis. 2014; 769:29-33.

30. Mlynarcikova A, Nagyova E, Fickova M and Scsukova S. Effects of selected endocrine disruptors on meiotic maturation, cumulus expansion, synthesis of hyaluronan and progesterone by porcine oocyte-cumulus complexes. Toxicology in Vitro. 2009; 23:371-377.

31. Nakano K, Nishio M, Kobayashi N, Hiradate Y, Hoshino Y, Sato E and Tanemura K. Comparison of the effects of BPA and BPAF on oocyte spindle assembly and polar body release in mice. Zygote. 2016; 24:172-180.

32. Ferris J, Favetta LA and King WA. Bisphenol A Exposure during Oocyte Maturation in vitro Results in Spindle Abnormalities and Chromosome Misalignment in Bos taurus. Cytogenetic and Genome Research. 2015; 145:50-58.

33. Can A, Semiz O and Cinar O. Bisphenol-A induces cell cycle delay and alters centrosome and spindle microtubular organization in oocytes during meiosis. Molecular Human Reproduction. 2005; 11:389-396.

34. Fan HY and Sun QY. Involvement of mitogen-activated protein kinase cascade during oocyte maturation and fertilization in mammals. Biology of Reproduction. 2004; 70:535-547.

35. Singh S and Li SS. Epigenetic effects of environmental chemicals bisphenol A and phthalates. International Journal of Molecular Sciences. 2012; 13:10143-10153. 
36. Trapphoff $\mathrm{T}$, Heiligentag $\mathrm{M}$, El Hajj $\mathrm{N}$, Haaf $\mathrm{T}$ and Eichenlaub-Ritter U. Chronic exposure to a low concentration of bisphenol A during follicle culture affects the epigenetic status of germinal vesicles and metaphase II oocytes. Fertility and Sterility. 2013; 100:1758-1767 e1751.

37. Susiarjo M, Sasson I, Mesaros C and Bartolomei MS. Bisphenol a exposure disrupts genomic imprinting in the mouse. PLoS Genetics. 2013; 9:e1003401.

38. Tilly JL. Commuting the death sentence: how oocytes strive to survive. Nature Reviews Molecular Cell Biology. 2001; 2:838-848.

39. Hutt KJ. The role of BH3-only proteins in apoptosis within the ovary. Reproduction. 2015; 149:R81-89.
40. Agarwal S, Tiwari SK, Seth B, Yadav A, Singh A, Mudawal A, Chauhan LK, Gupta SK, Choubey V, Tripathi A, Kumar A, Ray RS, Shukla S, et al. Activation of Autophagic Flux against Xenoestrogen Bisphenol-A-induced Hippocampal Neurodegeneration via AMP kinase (AMPK)/Mammalian Target of Rapamycin (mTOR) Pathways. The Journal of Biological Chemistry. 2015; 290:21163-21184.

41. Srivastava S, Gupta P, Chandolia A and Alam I. Bisphenol A: a threat to human health? Journal of Environmental Health. 2015; 77:20-26.

42. Agarwal A, Gupta S and Sharma R. Oxidative stress and its implications in female infertility - a clinician's perspective. Reproductive Biomedicine Online. 2005; 11:641-650. 